\title{
I TEMPI VERBALI DEL MODO INDICATIVO NEL DIALETTO ISTROVENETO DEL LITORALE SLOVENO
}

\author{
Suzana Todorović, Facoltà di Studi educativi dell'Università del Litorale, \\ suzana.todorovic@upr.si
}

\subsection{2/fII.30.2020.16 \\ UDK 811.162.4'28}

Nel presente contributo ${ }^{1}$ ci proponiamo di presentare l'impiego dei tempi verbali istroveneti del modo indicativo. Il lessico istroveneto (attinente all'area istriana nordoccidentale) è riportato in otto dizionari dialettali, ovvero Dizionario storico fraseologico etimologico del dialetto di Capodistria (Giulio Manzini e Luciano Rocchi, 1995), Dizionario del dialetto capodistriano (Dino Parovel, 2006), Voci della parlata isolana nella prima metà di questo secolo (Antonio Vascotto, 1987), Vocabolarietto del dialetto isolano (Antonio Delise, 2006), Dizionario del dialetto isolano: raccolta di parole e modi di dire della parlata isolana di ieri, di oggi e, forse, di domani (Silvano Sau, 2009), Le perle del nostro dialetto (Ondina Lusa e Marino Bonifacio, 2004, 2010, 2012). In alcuni dizionari gli autori riportano altresì una selezione di elementi grammaticali senza fornire un puntuale quadro grammaticale delle parlate prese in esame; oltre a ciò, non sempre vi è rispondenza tra gli elementi grammaticali riportati e quelli rilevati sul campo durante le nostre ricerche, essendo (stati) gli autori dei dizionari perlopiù amanti della parola dialettale e non linguisti o dialettologi.

Le regole d'uso dei tempi verbali istroveneti (presente, imperfetto, futuro semplice, passato prossimo, imperfetto, trapassato prossimo, futuro anteriore) sono state definite mediante materiali testuali registrati sul campo durante le inchieste dialettali effettuate in sette punti d'inchiesta istroveneti. Tutti i testi ricavati sul campo sono stati corredati da traduzioni in italiano.

Per ogni tempo verbale riportiamo le coniugazioni dei verbi ausiliari 'eser 'essere' e ver 'avere' e le coniugazioni dei verbi regolari con infinito in -ar o - a (Pirano); - er (Capodistria), - $e$ (Isola) o - $i$ (Pirano e Strugnano); -ir (Capodistria, Isola) o -i (Pirano). Nel caso dei tempi verbali composti è stata messa in rilievo la loro modalità di formazione.

\footnotetext{
${ }^{1}$ Per la stesura dei testi si è utilizzato il sistema di caratteri ZRCola elaborato dal prof. Peter Weiss del Centro di ricerca scientifica dell'Accademia Slovena delle Scienze e delle Arti - ZRC SAZU.
} 
Parole chiave: modo indicativo, tempi verbali, dialetto istroveneto, Istria slovena, dialettologia

\section{II dialetto istroveneto parlato nell'Istria slovena}

L'istroveneto è un dialetto di matrice veneta che iniziò ad estendersi lungo le coste istriane a partire dal $X$ secolo - in questo periodo la Serenissima intratteneva con le cittadine istriane intensi e amichevoli rapporti economici. II dominio politico e culturale di Venezia si consolidò appena nel XIII secolo, all'epoca dell'assoggettamento di Capodistria, Isola e Pirano, rispettivamente negli anni 1279, 1280 e 1283. Nel 1420 la Serenissima istituì sull'intero territorio istriano un sistema amministrativo e giuridico unitario. Gli istriani di origini romanze accettarono senza indugio la nuova lingua (molto probabilmente già in epoca precoce, nel $\mathrm{X} \circ \mathrm{XI}$ secolo), ${ }^{2}$ lasciando cadere nell'oblio l'unico idioma istriano autoctono - I'istrioto, espansosi in Istria all'epoca della romanizzazione. Il dialetto istrioto è tuttora vivo soltanto a Valle, Rovigno, Fasana, Gallesano, Dignano d'Istria e Sissano.

A subire fortemente l'influsso e la totale preponderanza della Serenissima ${ }^{3}$ furono pure gli altri idiomi neolatini - il dalmatico (XI-XV sec.), il friulano di Muggia e Trieste (XIX sec.) ed il veglioto, parlato sull'isola di Veglia (XIX sec.) (Filipi, 1994: 29).

Fino alla calata degli Slavi (attorno all'VIII secolo) l'istrioto era I'unica lingua parlata in Istria; I'insediamento della nuova popolazione fece progressivamente emergere quella dicotomia etnica tipica dell'Istria, con le città dal carattere etnico romanzo, da un lato, e la campagna dal prevalente elemento slavo, dall'altro (Todorović 2019, 19; Mihelič 2007, 31; 39-40; Darovec 2008, 151). La popolazione di origine slava (successivamente slovena) che già in epoca molto antica si stabilì nei centri urbani non vi sviluppò mai, tuttavia, idiomi dialettali propri. Gli istriani di provenienza slava diedero origine al dialetto istrosloveno articolandolo in due varietà subdialettali - quella risana e quella savrina, adoperate tutt'oggi attivamente nel profondo entroterra alle spalle di Capodistria, Isola e Pirano.

Fino alla fine della Seconda guerra mondiale il dialetto istroveneto era l'idioma più diffuso in Istria, ricoprendo il ruolo di koinè di tutti gli istriani. Mentre però la popolazione istroveneta urbana parlò fino alla fine della Seconda guerra mondiale un'unica lingua, gli istriani

\footnotetext{
${ }^{2}$ Cfr. Filipi 1994, 29.

3 La dominazione veneziana è tutt'oggi rilevabile nell'architettura e nella cultura delle città istriane e dalmate.
} 
dell'entroterra erano per lo più bilingui. Ne deriva che gli italiani oggi residenti in località bidialettali abbiano una competenza bilingue, ma di tipo passivo; i più anziani di nazionalità slovena sono invece in grado di esprimersi in entrambi i dialetti, dato che in passato conoscere la koinè istriana era importante per poter comunicare con la popolazione monolingue dei centri urbani costieri e di Trieste. In epoca antecedente all'amministrazione italiana e al relativo processo di sistematica italianizzazione erano però rari i casi di istriani che parlassero anche I'italiano letterario allora invalso (Todorović 2019, 23).

Con la capitolazione dell'Italia, l'Istria viene annessa alla Repubblica popolare federativa di Jugoslavia (FNRJ). ${ }^{4}$ Dopo la Seconda guerra mondiale gran parte degli abitanti di origini italiane dell'area urbana si trasferì in Italia o altrove nel mondo. Allora ebbe inizio il progressivo afflusso di persone provenienti dalle campagne, dalle regioni interne della Slovenia e dalle altre ex repubbliche jugoslave, che si accentuò negli anni Sessanta e Settanta del XX secolo con la nascita dei primi grandi stabilimenti industriali, che avevano bisogno di molta manodopera. Tutto ciò portò alla formazione di una serie di differenze nella struttura sociale, culturale ed etnica della popolazione (Žitko 2011, 30-31).

Dall'ultimo censimento generale della popolazione slovena del 2002 si rileva che l'italiano è la lingua madre di 3.762 cittadini sloveni, di cui il 76 percento vive a Capodistria (e Ancarano), Isola e Pirano, ovvero 1.059 a Capodistria, 620 a Isola e 1.174 a Pirano. Per il 31,7 percento dei cittadini di madrelingua italiana la lingua parlata in famiglia coincide con la lingua madre, il 30,7 percento usa in famiglia lo sloveno, mentre il 35 percento parla entrambe le lingue (Šircelj 2002, 107).

Nel corso delle ricerche condotte sul dialetto istroveneto (Todorović 2017, 2019) si è constatato che la maggior parte degli italofoni in Istria si identifica infatti con l'istroveneto, che ancora oggi rappresenta la lingua madre degli appartenenti alla comunità nazionale italiana. Con l'esodo dei cittadini di origine romanza nell'immediato dopoguerra questo dialetto ha perso il 90 per cento dei suoi parlanti, riducendosi al solo uso nell'ambito della minoranza italiana.

Con riferimento all'Istria slovena, l'area d'uso dell'istroveneto è stata individuata sulla base delle ricerche dialettologiche effettuate in più punti $d^{\prime}$ inchiesta istrosloveni e istroveneti, ${ }^{5}$ indagando ogni volta soprattutto tra i residenti locali della vecchia generazione - l'uso del

\footnotetext{
${ }^{4}$ Successivamente (nel 1945) Repubblica Socialista Federale di Jugoslavia.

${ }^{5}$ Condotte dall'autrice dell'articolo.
} 
dialetto in passato e al giorno d'oggi, i flussi migratori in entrata e altri avvenimenti legati alla dimensione sociale che hanno potenzialmente influito anche sul dialetto delle località in questione. ${ }^{6}$ I dati così reperiti sono stati confermati da più abitanti del luogo e messi in correlazione ai vari eventi storici. Sulla scorta di quanto sopra si è perciò constatato che gli istriani parlano in istroveneto a Pirano, Portorose, Lucia, Strugnano, Sezza, Sicciole, Parezzago, Dragogna, Isola (Dobrava presso Isola, Jagodje), Bossamarino, Campel, Salara, Capodistria, San Canziano, Bertocchi (Prade), Valmarin (parte dell'abitato di Scoffia di Sotto/Spodnje Škofije), Ancarano, Barisoni, Colombano, Cerei, Premanzano e Crevatini. A Bertocchi, Crevatini, Cerei, Premanzano e Dragogna si rileva l'uso di entrambi i dialetti istriani da parte degli abitanti locali, dunque sia dell'istroveneto che dell'istrosloveno, nelle restanti località dell'Istria slovena invece solo il secondo (Todorović 2019: 90).

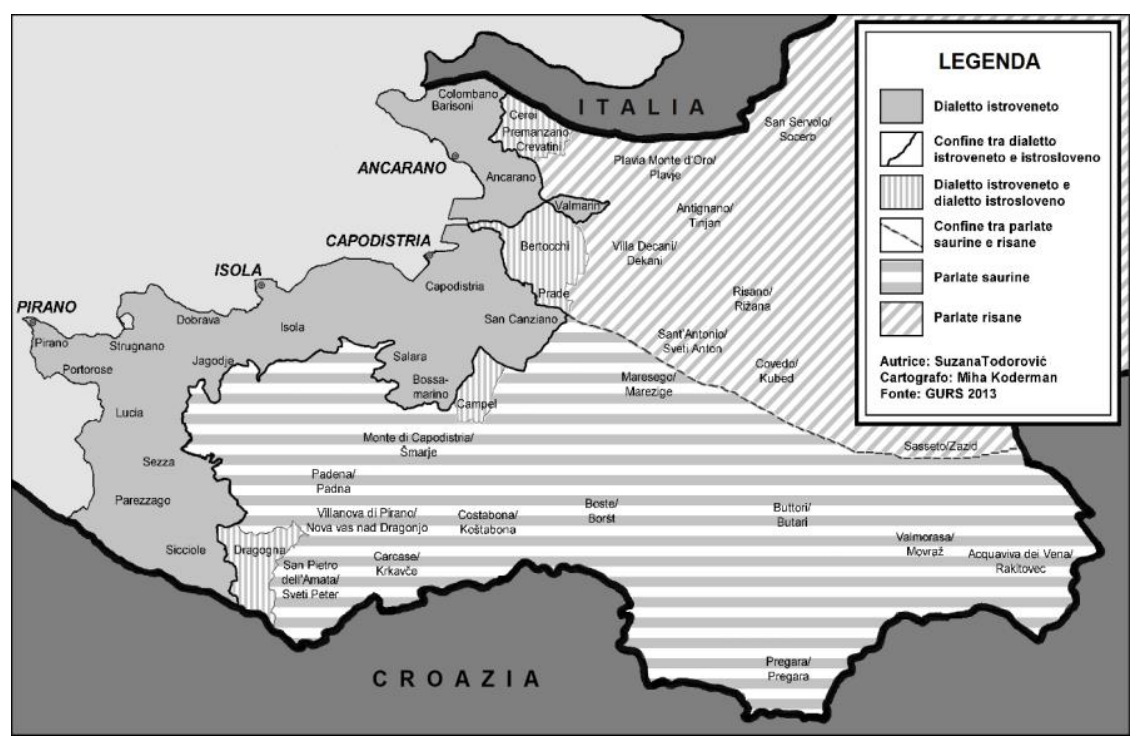

Cartina 1: L'area d'uso del dialetto istroveneto in Slovenia (Todorović 2019: 127)

Negli ultimi cinquant'anni il dialetto istroveneto ha perso le peculiarità dialettali in base alle quali in passato i suoi parlanti distinguevano l'una dall'altra le diverse varietà; oggigiorno esse sono riscontrabili soprattutto nella lingua degli istrovenetofoni più anziani, che adoperano l'idioma del luogo natio con familiari e concittadini

${ }^{6}$ Cfr. Todorović, 2015a: 52-53. 
(compaesani), mentre con gli altri appartenenti alla comunità nazionale italiana (provenienti soprattutto da località istriane croate) adoperano una variante di istroveneto più uniformata. I parlanti più giovani, generalmente meno conservativi nell'uso del dialetto, introducono nel discorso dialettale espressioni slovene e italiane adattandole foneticamente e morfologicamente al loro dialetto.

\section{Il metodo di ricerca adoperato nel rilevare l'uso del modo indicativo}

Per ottenere un quadro generale sull'impiego dei tempi verbali del modo indicativo fra i dialettofoni istroveneti ci siamo avvalsi dei dati acquisiti durante diverse ricerche dialettologiche svolte ${ }^{7}$ in sette punti d'inchiesta istroveneti, ovvero a Capodistria, Isola, Pirano, Strugnano, Sicciole, Crevatini e Bertocchi. I parlanti sono stati incoraggiati a produrre testi orali pertinenti al passato, all'attualità e all'avvenire. I racconti dialettali sono stati registrati e in un secondo momento trascritti e ordinati. Sulla scorta dei testi dialettali così ottenuti si è potuto procedere con un'analisi dettagliata dell'uso del modo indicativo. $^{8}$

Per i singoli tempi verbali istroveneti presentiamo il loro ambito d'uso, osservandoli (in più occasioni) in un'ottica contrastiva con i tempi verbali italiani. Tutte le frasi, nonché i testi dialettali, sono corredati di traduzione in lingua italiana.

Per la trascrizione delle produzioni in dialetto abbiamo utilizzato i grafemi della scrittura fonetica italiana, ovvero $b, d, f, g, j, k$, $l, m, n, p, r, t, v, a, e, e, i, o, o, u$, aggiungendo alcuni fonemi specifici come:

$\dot{c}$ - affricata prepalatale, fonema intermedio tra $\check{c}$ e ć, ad es. cor 'prendere'; $\breve{g}$ - affricata prepalatale sonora, ad es. 'oği 'oggi'; a semivocale, vocale centrale neutrale, ad es. an'dar 'andare'; $y-$ fricativa velare sonora, ad es. bo'teva 'negozio'; $I$ - I palatale, ad es. paśtila 'pastiglia'; $n$ - $n$ palatale, ad es. ma'ńar 'mangiare'; $\dot{s}-$ fricativa alveolare, fonema intermedio fra $s$ e $\breve{s}$, ad es. ka'piso 'capisco'; $\dot{z}-$ fricativa alveolare, fonema intermedio fra $z$ e $z$, ad es. 'kużo 'cucio'; $w-$ semivocale $w$, ad es. kwel 'quello'; c - affricata dentale sorda (solamente a Crevatini e Bertocchi), ad es. cer'kar 'cercare'; 3 - affricata dentale sonora (solamente a Crevatini e Bertocchi), ad es. $3 a$ 'già'.

\footnotetext{
${ }^{7}$ In modo autonomo da parte dell'autrice del presente contributo.

${ }^{8}$ Nell'ambito delle inchieste sono stati studiati, inoltre, altri elementi lessicali e grammaticali, nonché il lessico dialettale.
} 
Il segno indicante la posizione dell'accento (' ) si colloca davanti alla sillaba tonica, ma è stato omesso in caso di parole monosillabe.

\section{II modo indicativo nel dialetto istroveneto}

II dialetto istroveneto contempla, ${ }^{9}$ come l'italiano letterario, sette modi verbali - di cui quattro modi finiti (indicativo, congiuntivo, condizionale, imperativo) e tre modi indefiniti (infinito, participio, gerundio).

Mentre nell'italiano letterario il modo indicativo si articola in otto tempi verbali (presente, imperfetto, passato prossimo, passato remoto, trapassato prossimo, trapassato remoto, futuro semplice e futuro anteriore), il dialetto istroveneto ne conosce sette, essendo il passato prossimo usato a scapito ${ }^{10}$ del passato remoto.

\section{Presente}

Con il tempo presente i dialettofoni istroveneti comunicano situazioni che si svolgono al momento dell'enunciazione, ad esempio 'Deśo son 'każa e śpeto mi ma'ri kel 'veńi dal la'vor. 'Adesso sono a casa e aspetto mio marito che venga ${ }^{11}$ dal lavoro.' (Crevatini, N. B.).

'Oği no yo 'colto ni'anka la paś'tila e me djol 'kome 'jeri. 'Oggi non ho neanche preso la pastiglia e mi fa male come ieri.' (Isola, A. M.)

II presente istroveneto indica pure le consuetudini (anche usanze) del soggetto, ad esempio

De ma'tina 'vado in bo'teva, me 'ćovo el ka'rẹto e kon kwel ka'mino 'mejo. In 'każa pjan pjan 'faso da 'sọla, 'solo 'devo sen'tarme, że ku'si, kos te vol ... 'Di mattina vado in bottega, vado con il carrello, che con quello cammino meglio. In casa piano piano sbrigo le mie faccende da sola, solo che ogni tanto devo sedermi, così è, cosa vuoi farci ... (Isola, A. M.).

Mi yo el me do'tọr ğene'rale kwa 'Iżola. Ko ke 'vado a ċor medi'żine o me djol kwal'kośa 'ćerko de parl'lar żlo'ven, ma lu

\footnotetext{
${ }^{9}$ Cfr. Dardano e Trifone 2013, 311.

${ }^{10}$ Tratto caratteristico di tutti i dialetti italiani settentrionali.

${ }^{11}$ Nella frase italiana si richiede l'uso del congiuntivo presente, solitamente sconosciuto nel dialetto istroveneto.
} 
me ris'pondi in ita'lan, par'ke ve inte'resa de par'lar. 'Io ho il medico di base qui a Isola. Quando vado a prendere le medicine o mi fa male qualcosa cerco di parlare sloveno, ma lui mi risponde in italiano, perché gli interessa parlare in lingua.' (Isola, A. M.).

I Ita'ĺani kwa a 'Iżola i że 'anka del 'Istrija. Par'lẹmo 'senpre in dija'leto, pe'ro 'vẹmo un 'altra ka'dẹnsa de par'lar. Ma se se ka'pisi lo s'teśo. Mi 'parlo in dija'leto kon 'duti, mi 'parlo 'kome $k e$ so. Kon 'poki 'parlo żlo'ven. 'Gli italiani qui a Isola vengono anche dall'Istria. Parliamo sempre in dialetto, però abbiamo un'altra cadenza. Comunque ci capiamo. lo parlo in dialetto con tutti, parlo come so. Solo con pochi mi metto a parlare in sloveno.' (Isola, A. M.)

Con il tempo presente gli istriani di origine romanza indicano pure avvenimenti che devono ancora realizzarsi, ad esempio

'Deśo te 'digo 'dove ke 'vado do'mam (Pirano). 'Adesso ti dico dove vado domani.'

(Capodistria, P. F.)

'Tẹnto, ke ye 'divo mi tu 'mama kos ke te 'fasii! 'Attento che lo vado a dire a tua madre, quello che combini!' (Isola, D. B.).

Il presente (nella funzione del cosiddetto presente storico) viene adoperato anche (al posto del passato prossimo o dell'imperfetto) per raccontare un evento passato concluso, ad esempio

El me 'vara, sta 'żito, me 'vara 'naltra 'volta, se 'ğira e me 'diżi: "Ma 'ela no la ka'pisi 'ńente!» 'Lui mi guarda, sta zitto, mi guarda un'altra volta, si gira e mi dice: "Ma lei non capisce niente!»' (Bertocchi, B. C.).

II presente può indicare il verificarsi di proprietà permanenti o eterne (il cosiddetto presente atemporale), formulate come "eterne verità", proverbi ecc., ad esempio

Pju 'bela ke la vol far, pju b'ruta la fa. 'Più bella vuole apparire, più brutta appare.' (Isola, E. D.)

Se ti vol sta ben 'mańa 'poko e 'dormi ben. 'Se vuoi stare bene mangia poco e dormi bene.' (Pirano, M. B.). 
Coniugazione dei verbi ausiliari essere ('eser) e avere (ver) al presente indicativo

Il verbo ausiliare ver 'avere' viene spesso sostituito dal verbo di origine triestina italiana $v a$ 'ver, che si manifesta soprattutto nella prima e nella seconda persona plurale.

Le parlate bertossana (Bertocchi) e crevatinese (Crevatini) presentano una realizzazione più aperta delle vocali toniche $e$ ed $o$, ad esempio noj'altri 'semo 'noi siamo', mi son 'io sono', ti te son 'tu sei', noj'altri 'vemo 'noi abbiamo'; tutti gli altri idiomi prevedono talvolta una realizzazione marcatamente chiusa, ad esempio a Capodistria noj'altri 'vẹmo 'noi abbiamo', mi sọn 'io sono'.

\begin{tabular}{|c|c|c|}
\hline & 'eser 'essere' & $(v a)^{\prime}$ ver 'avere' \\
\hline $\mathrm{mi}$ & sọn & yo \\
\hline ti $(\mathrm{te}, \mathrm{ti})^{12}$ & sọon $(\dot{z} e)^{13}$ & va \\
\hline lu (el) & $\dot{z} e$ & $y a$ \\
\hline 'ela (la) & zie & va \\
\hline noj'altri & 'sẹmo & 'vẹmo, va'vẹmo \\
\hline voj'altri & se & ve, ya've \\
\hline 'lori (i) & że & va \\
\hline 'lore (le) & $\dot{z} e$ & va \\
\hline
\end{tabular}

\section{Coniugazione dei verbi regolari in -are, -ere e -ire}

Nei verbi (regolari) la desinenza dell'infinito -ar risulta uniforme in tutte le varietà istrovenete ad eccezione del vernacolo istroveneto di Pirano, ove è adoperata la desinenza - $a$, ad esempio a Isola ma'ńar, a Pirano ma'ńar 'mangiare', kan'ta 'cantare', la'va 'lavare', mis'ja 'mescolare' ...

${ }^{12}$ Nel dialetto preso in esame è tipico l'uso del doppio pronome personale nella seconda e terza persona singolare, nonché nella terza persona plurale, ad esempio 'Ẹla la że inferm'jera., 'Ella è infermiera.', La 'ćakola śaj. 'Ella chiacchiera molto.', ma spesso viene usata solo la seconda parte del pronome, ad esempio El spar'laca. 'Egli spettegola.'.

${ }^{13}$ Forma in disuso. 
L'infinito in -er si presenta negli idiomi istroveneti con diverse realizzazioni, ad esempio a Capodistria, Isola, Sicciole, Crevatini e Bertocchi 'eser 'essere', bever 'bere', a Isola 'ese, 'beve, a Pirano e Strugnano 'esi, 'bevi.

\begin{tabular}{|l|l|l|l|}
\hline & -ar & -er & $-i r$ \\
mi & ma'ńar 'mangiare' & 'verżer 'aprire' & ku'żir 'cucire' \\
ti (te, ti) & 'mańo & 'verżo & 'kużo \\
lu (el) & 'mańa & 'verżi & 'kużi \\
'ela (la) & 'mańa & 'verżi & 'kużi \\
noj'altri & mań'ẹmo & 'verżi & 'kużi \\
voj'altri & ma'ńe & ver'żẹmo & $k u$ 'żimo \\
'lori (i) & 'mańa & ver'żẹ & $k$ 'żi \\
'lore (le) & 'mańa & 'verżi & 'kużi \\
\hline
\end{tabular}

Presentiamo di seguito le coniugazioni dei verbi modali:

do'ver 'dovere': 'devo, 'deve, 'deve, do'vẹmo, do've, 'dove po'dẹr 'potere': 'posio, pol, pọl, po'dẹmo, po'de, pọl vo'lẹr 'volere' 'vojo, vọl, vol, vo'lemo, vo'le, vọl $\dot{s} a$ 'ver 'sapere': $\dot{s}{ }^{14}{ }^{14} \dot{s} a, \dot{s} a, \dot{s} a$ 'vẹmo, śa've, śa

II verbo modale do'vẹr 'dovere' è attestato nel dialetto attuale (cfr. Todorović 2016, 230) per effetto dell'influsso dell'italiano standard. In passato, per esprimere necessità (nel dialetto più antico) si soleva infatti usare il verbo ver 'avere' con la preposizione de, ad esempio a Capodistria e Isola Go de (a)n'dar (a) 'każa. 'Devo andare a casa'.

\section{Imperfetto}

L'imperfetto istroveneto indica fondamentalmente la simultaneità rispetto ad un momento passato, ad esempio

${ }^{14}$ Anche: 'śapjo. 
Ko son an'dada cer'kar la'vor, do'vevo par'lar żlo'ven ... 'Quando sono andata a cercare lavoro dovevo parlare sloveno ...' (Bertocchi, B. K.)

$K$ 'wando an'dajmo 'fora de 'fabrika 'tante 'volte se fer'majmo un 'poko. 'Una 'sera 'jero kon mi ma'ri e mi so'rela 'kola pi'ńata la że vi'ńuda. La me ya da la pi'ńata in 'testa. 'Spesso, uscendo dalla fabbrica, ci fermavamo un po'. Una sera ero con mio marito e mia sorella è venuta con la pentola. E me l'ha sbattuta in testa.' (Isola, E. D.).

I dialettofoni istroveneti usano questo tempo verbale anche per descrivere azioni ripetute e continuative nel passato, ad esempio 'Ońi di'meneva 'jera el 'balo al Ari'yoni. An 'dajmo ba'lar 'anka in Ka'fe 'Menis. La an 'dava 'solo i s'jori. 'Jera un bel lo'kal. Un lo'kal fin. Me ma'ri va'vẹva un pa'rẹte ke 'jera kamer'jer e a ne fa'sevva an'dar. 'Ogni domenica c'era il ballo all'Arrigoni. Andavamo a ballare anche al caffè Menis. $\mathrm{Ci}$ andavano soltanto $\mathrm{i}$ signori. Era un bel locale. Un locale elegante. Mio marito aveva un parente che faceva il cameriere e ci faceva entrare.' (Isola, E. F.).

Con l'imperfetto indicativo istroveneto i parlanti designano stati di cose, segnalano azioni incompiute nel passato ecc., ad esempio

I iżo'lani in'dava in kan'pańa, 'duto kwa in'torno 'Iżola 'jera kan'panje, Ba're, Servi'ńan, Preva'vọr, Monte'kalvo, 'Kanola. In'dava de 'matina kol ażi'nel e la 'sera i tor'nava. 'Altri Iżo'lani lavo'rava in 'fabrika o 'jera peska'dọi. 'Jera 'anka i si'ńori kwa a 'Izola, 'jera do'tori, 'jera avo'kati e 'duti i par'lava el 'nostro iżo'lan.

'Gli isolani andavano nei campi, qui intorno c'erano solo terreni Barè, Cervignano, Pregavor, Monte Calvo, Cànola. Partivano di mattina con l'asinello e tornavano di sera. Gli altri che abitavano a Isola lavoravano in fabbrica o facevano i pescatori. Qui a Isola c'erano anche dei signori, erano dottori, avvocati e parlavano tutti nella nostra parlata locale.' (Isola, A. M.)

In Ampe'lea le ve'ńiva su daj 'monti. E 'kapo 'jera p'ropjo iżo'lan e par'lava ita'lan, noj par'lavimo in iżo'lan, 'lore le par'lava in su dija'leto, ma se se ka'piva. 'Mi se po'devo par'lavo 'senpre el me dija'leto. 'Jera 'certe 'anka kwa dej 'monti, 'una la 'jera de Ma'reżeyo, ke mi ve par'lavo 'anka un 'poko in żlo'ven k'walke 
'volta 'certe e 'lore le rispon'deva in ita'lan. Le vo'leva 'kome vici'narse. E 'dopo se ran'ğavimo lo s'tẹso. Se kapi'vimo. Mi 'parlo 'anka un 'poko de żlo'ven, ma no yo k'wẹla pro'nunća. 'Venivano all'Ampelea dai monti. Il capo era un italiano e parlava italiano, noi isolano, loro ognuna nel proprio dialetto, ma ci si capiva. Appena potevo, parlavo nel mio dialetto. Con alcune che erano qui dei monti, una era di Maresego (Marezige), ogni tanto parlavo un po' in sloveno e loro mi rispondevano in italiano. Volevano essermi più vicine. Per cui poi in un modo o nell'altro ce la facevamo. Io un po' parlo sloveno, ma non ho quella pronuncia.' (Isola, A. M.).

Con l'ausilio dell'imperfetto i parlanti narrano e descrivono azioni svoltesi nel passato, ad esempio

Ko 'jero 'pič i fjoj żjo'vava in 'modo di'verśo de 'oži. 'Ońi 'žorno 'dopo s'kola żo'vajmo da'vanti a s'kola. Żo'vajmo a 'korerse d'rijo, a s'konderse e ko le 'vave. In televiż'jon var'davo Goldrake, Heidi, L'ape Maia e Super Gulp. In is' 'ta an'dajmo a far el 'bańo in Belve'der. Śaj 'volte żo'yavo 'anka in 'orto. Me pja'żẹva ka'pir 'kome func'jona le 'robe, saj 'volte ver'żevo el żo'vatolo per 'veder 'kome el ga el meka'niżmo. Me ri'kordo de 'una 'veća 'radjo a 'valvole, mi la var'davo d'rento per ri'var a 'kapir 'kome ven 'fora el sọn, me 'jero 'meso in 'testa de kostru'ir 'una, ma no tro'vavo ni'sun ke sa'veva 'kome. K'walke 'ano 'dopo la go kostru'ida, la 'mija p'rima 'radjo a kris'talo. 'Quand'ero piccolo i bambini giocavano in modo diverso rispetto ad oggi. Ogni giorno, dopo le lezioni, giocavamo davanti alla scuola. Giocavamo a rincorrerci, a nascondino e con le biglie. Alla televisione seguivo Goldrake, Heidi, L'ape Maia e Super Gulp. In estate andavamo a fare il bagno sotto Belvedere. Molto spesso giocavo anche in orto. Mi piaceva capire come funzionassero gli oggetti, molto spesso aprivo i giocatoli per vedere come fosse il meccanismo. Mi ricordo di una vecchia radio a valvole, ma la guardavo all' interno per riuscire a capire come uscisse il suono, mi ero messo in testa di costruirne una, ma non trovai nessuno che sapesse come si fa. Qualche anno più tardi l'ho costruita, la mia prima radio a cristallo.' (Capodistria, P. R.).

Con questo tempo verbale i parlanti del dialetto formulano frasi ipotetiche relative al periodo ipotetico dell'irrealtà, con 
riferimento al passato. In esse si osserva l'uso dell'imperfetto indicativo sia nella frase reggente che in quella dipendente, ${ }^{15}$ ad esempio

'Jera 'mejo se (lu) el me di'żẹvi p'rima. 'Era meglio se me lo diceva prima./Sarebbe stato meglio se me lo avesse detto prima.' (Capodistria, A. C.).

Coniugazione dei verbi ausiliari essere ('esंer) e avere (ver) all'imperfetto indicativo

\begin{tabular}{|l|l|l|}
\hline $\mathrm{mi}$ & 'eser 'essere' & $($ va)'ver 'avere' \\
$\mathrm{ti}(\mathrm{te}, \mathrm{ti})$ & 'jero & va'vẹvo, 'vẹvo \\
lu (el) & 'jeri & va'vẹvi, 'vẹvi \\
'ela (la) & 'jera & va'vẹva, 'vẹva \\
noj'altri & 'jerimo & va'vẹva, 'vẹva \\
voj'altri & 'jeri, 'jerivi & va'vẹvimo, 'vevimo \\
'lori (i) & 'jera & va'vẹvi, 'vẹvi \\
'lore (le) & 'jera & va'vẹva, 'vẹva \\
\hline
\end{tabular}

Coniugazione dei verbi regolari in -are, -ere e -ire all'imperfetto indicativo

\begin{tabular}{|c|c|c|c|}
\hline & $\begin{array}{l}\text {-ar } \\
\text { pre'yar 'pregare' }\end{array}$ & $\begin{array}{l}\text {-er } \\
\text { le'ǧẹr 'leggere,16 }\end{array}$ & $\begin{array}{l}\text {-ir } \\
\text { sin'tir 'sentire' }\end{array}$ \\
\hline $\mathrm{mi}$ & pre'yavo & le'ğẹvo & sin'tivo \\
\hline ti (te, ti) & pre'yavi & le'ğẹvi & sin'tivi \\
\hline lu (el) & pre'vava & le'ğẹva & sin'tiva \\
\hline 'ela (la) & pre'vava & le'ğeveva & sin'tiva \\
\hline noj'altri & pre'vajmo & le'ğẹvimo $^{17}$ & sin'tivimo \\
\hline voj'altri & pre'yavi & le'ğẹvi & sin'tivi \\
\hline 'lori (i) & pre'vava & le'ğẹva & sin'tiva \\
\hline 'lore (le) & pre'yava & le'ğeveva & sin'tiva \\
\hline
\end{tabular}

${ }^{15}$ Come nell'italiano colloquiale.

${ }^{16}$ Anche: 'leżer.

${ }^{17}$ Anche: le'ğejmo. 
Per la prima persona plurale dei verbi i dialettofoni conoscono perlopiù due desinenze: - avimo, ad esempio preva'vimo 'pregavamo', e -ajmo, ad esempio pre' yajmo; I'uso della prima desinenza si rileva nel dialetto più recente, sottoposto ad un influsso sempre più consistente dell'italiano standard; la desinenza -ajmo, invece, è una peculiarità conservata dai dialettofoni più anziani (di'żevimo e di'żejmo 'dicevamo', kan'tavimo e kan'tajmo 'cantavamo', pron'tavimo e pron'tajmo 'preparavamo' ...).

\section{Futuro semplice}

Nel vernacolo istroveneto, con il tempo futuro i parlanti annunciano avvenimenti che devono ancora svolgersi o che si realizzeranno in futuro, ad esempioKo te trova'ra un la'vor tel viva'ra 'mejo. 'Quando troverai un lavoro vivrai meglio.' (Bertocchi, B. K.)

Te son saj b'rava de lavo'rar, 'deśo te va'ra una 'papa pju 'bona. 'Lavori molto bene, per cui avrai una paga migliore.' (Saredo, A. T.)

Koś te fa'ra par 'sena, mi 'pesii f'reśko, p'rimo 'tajo de ra'dicio e po'leta ... ma ke bon, ma ke bon ... 'Cosa prepari per cena, io pesce fresco, radicchietto e polenta ... oh che bontà, oh che bontà ...' (Saredo, A. T.)

Śta 'sera ke ma'ńada, ko veńa'ra 'każa 'Bepi ... 'Che mangiata stasera, quando tornerà a casa Bepi ...' (Saredo, A. T.).

Coniugazione dei verbi ausiliari essere ('eser) e avere (ver) al futuro semplice

\begin{tabular}{|c|c|c|}
\hline & 'eśer 'essere' & ( $(\mathrm{a} a)^{\prime}$ 'ver 'avere' \\
\hline $\mathrm{mi}$ & $\dot{s} a^{\prime} r o$ & va'ro \\
\hline ti (te, ti) & $\dot{s} a^{\prime} r a$ & va'ra \\
\hline lu (el) & $\dot{s} a ' r a$ & va'ra \\
\hline 'ela (la) & $\dot{s} a^{\prime} r a$ & va'ra \\
\hline noj'altri & śa'rẹmo & va'rẹmo \\
\hline voj'altri & sa're & va're \\
\hline 'lori (i) & $\dot{s} a{ }^{\prime} r a$ & va'ra \\
\hline 'lore (le) & $\dot{s} a^{\prime} r a$ & va'ra \\
\hline
\end{tabular}


Coniugazione dei verbi regolari in -are, -ere e -ire al futuro semplice

\begin{tabular}{|c|c|c|c|}
\hline & $\begin{array}{l}\text {-ar } \\
\text { bru'żar } \\
\text { 'bruciare' }\end{array}$ & $\begin{array}{l}\text {-er } \\
\text { pro'meter } \\
\text { 'promettere' }\end{array}$ & $\begin{array}{l}\text {-ir } \\
\text { dor'mir 'dormire' }\end{array}$ \\
\hline $\mathrm{mi}$ & bruża'ro & promete'ro & dormi'ro \\
\hline ti (te, ti) & bruża'ra & promete'ra & dormi'ra \\
\hline lu (el) & bruża'ra & promete'ra & dormi'ra \\
\hline 'ela (la) & bruża'ra & promete'ra & dormi'ra \\
\hline noj'altri & bruża'remo & promete'remo & dormi'remo \\
\hline voj'altri & bruża're & promete're & dormi're \\
\hline 'lori (i) & & promete'ra & dormi'ra \\
\hline 'lore (le) & bruża'ra & promete'ra & dormi'ra \\
\hline
\end{tabular}

\section{Passato prossimo}

Con il tempo passato prossimo indicativo i dialettofoni istroveneti descrivono avvenimenti conclusisi nel passato, ad esempio Nel 53 i va se'ra i b'loki; 'jera i b'loki se'raj, la de Śko'fije, no te po'devi in'dar 'oltre, Tri'jeste. E la 'ğente se va un 'poko ċa'pa pa'ura. Te sa, ki va'vevva pa'renti 'oltre e i di'żẹva: "Kwa i va se'ra i b'loki, no veda'remo pju ni'sun ..." 'Dopo że sta 'kome un epide'mija, 'duti i va komin'ća in'dar vija, pe'ro 'dopo że sta 'anka la 'ćeża ke va śaj influ'i su k'westo. Par'ke se a Tri'jeste no ve 'dava 'vito e a'loğo, no in'dava 'vija 'tanta 'ğente... Par'ke 'tanti i że in'da 'vija de kwa, a mo'rir i że in'daj su ... Kwa i va la'śa 'każe, kan'pańe, 'duto ... 'Dopo i b'loki i że staj 'verti, no so k'wanti 'meżi ke ya du'ra se'raj. 'Nel 1953 chiusero i valichi di frontiera; a Scoffie (Škofije) i valichi erano chiusi, non potevi passare e andare a Trieste. La gente si era un po' spaventata. Sai, chi aveva parenti là dall'altra parte diceva: "Qui hanno chiuso i valichi, non rivedremo più nessuno ...". Dopo c'è stata come un'epidemia, hanno iniziato tutti ad andarsene, ma poi anche la Chiesa ha pesato molto su questo. Se a Trieste non gli avessero offerto da mangiare e da dormire, non sarebbero partiti in così tanti. Molti sono andati via da queste parti, sono andati a morire su. Qui hanno abbandonato case, campi, tutto ... E poi hanno riaperto i blocchi, non so per quanto siano rimasti chiusi.'

(Isola, A. M.). 
II passato prossimo istroveneto è formato dal verbo ausiliare 'eser 'essere' oppure ver 'avere' al presente e dal participio passato del verbo. Le regole che determinano la scelta tra un ausiliare e l'altro sono le stesse pertinenti alla grammatica italiana ${ }^{18}$ (da notare I'uso antico dell'ausiliare ver 'avere' con il verbo sta(r) 'stare', ad es. yo sta, in italiano sono stato). Quando il passato prossimo è formato con l'ausiliare 'eser, la desinenza del participio deve concordare nel numero e nel genere con il soggetto, come accade nella lingua italiana, ad es. ausiliare essere + participio: Mi sọn vi'ńu (m.)., Mi sọn vi'ńuda (f.)., Lu a że vi'ńu., Ela la że vi'ńuda., 'Lori i że vi'nuuj., 'Lọre le że vi'ńude.; ausiliare ver + participio: Mi yo ma'ńa., 'Ela la va ma'ńa., 'Lori i 'vẹva ma'ńa., 'Lore le 'vẹva ma'ńa. Tale regola grammaticale si applica a tutti i tempi verbali composti (Todorović 2017, 223).

II participio passato istroveneto viene formato (come nella lingua italiana letteraria) dalla radice del verbo e dalla desinenza del participio passato in concordanza con il morfema del verbo all'infinito.

In piranese i morfemi del participio sono -ado, -udo e -ido, ${ }^{19}$ mentre nelle altre parlate venete dell'Istria slovena sono $-a,-u$ (-esto) o $-i$, in base alla desinenza del verbo all'infinito:

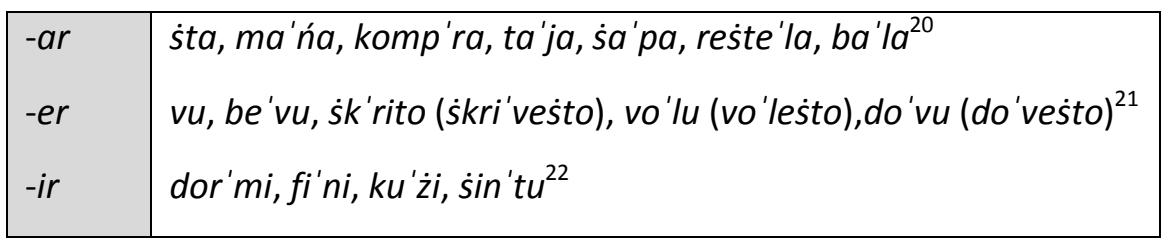

Dai racconti dialettali abbiamo estrapolato alcune forme di participio passato oramai obsolete, ma custodite ancora dai dialettofoni più anziani, ad esempio ( $\gamma a$ )'vẹsto (ver 'avere') e śa'vẹsto (śa'vẹr 'sapere'), pjan'żẹsto ( $p$ 'janżer 'piangere'), vo'lẹsto (vo'lẹr 'volere'), kre'dẹsto (k'reder 'credere'), pja'żẹsto ( $p$ 'jażer 'piacere'), do'vẹsto (do'vẹr 'dovere'). ${ }^{23}$

\footnotetext{
${ }^{18}$ Cfr. Todorović 2017, 223.

${ }^{19}$ Corrispondenti ai morfemi italiani -ato, -uto, -ito.

${ }^{20}$ Stato, mangiato, comprato, tagliato, zappato, rastrellato.

${ }^{21}$ Avuto, bevuto, scritto, voluto, dovuto.

${ }^{22}$ Dormito, finito, cucito, sentito.

${ }^{23}$ Cfr. Todorović 2017, 224.
} 


\section{Trapassato prossimo}

II trapassato indicativo istroveneto indica azioni anteriori rispetto ad un momento conclusosi nel passato, ad esempio

Mi disp'jażi ke no yo inpa'ra ben żlo'ven. 'Tanto mi disp'jażi. Ko son an'dada lavo'rar, par'lavo mal żlo'ven, par'ke noj a 'każa va'vemo par'la 'sempre ita'ĺan. 'Mi dispiace di non aver imparato bene lo sloveno. Mi dispiace molto. Quando sono andata a lavorare parlavo male lo sloveno, perché noi a casa usavamo/avevamo usato sempre l'italiano.'.

Questo tempo verbale è formato dal verbo 'eser 'essere' oppure ver 'avere' all'imperfetto e dal participio passato, ad esempio va'vejmo par'la 'avevamo parlato', va'vevo salu'da 'avevo salutato', 'jero an'dada 'ero andata', 'vẹvo ku'zi 'avevo cucito', 'vevo ba'la 'avevo ballato' ...

\section{Futuro anteriore}

Con questo tempo verbale i dialettofoni istroveneti descrivono avvenimenti anteriori rispetto ad un'azione futura (espressa con il futuro semplice). Il futuro anteriore istroveneto è composto dall'ausiliare 'eser 'essere' o ver 'avere' al tempo futuro e dal verbo al participio passato, ad esempio

Ko te pjanta'ra i pomo'dori, i 'miji śa'ra ża 'fati. 'Quando pianterai i pomodori, i miei saranno già cresciuti.' (Capodistria, P. R.)

Ko riva'rẹmo (a) Pola, 'Paolo va'ra ża prepa'ra el p'ransio. 'Quando arriveremo a Pola, Paolo avrà già preparato il pranzo.' (Isola, A. M.)

Ko riva'remo 'każa, vava'ra za pjo'vesto. 'Quando arriveremo a casa, avrà già piovuto/cominciato a piovere.' (Crevatini, N. B.).

\section{Conclusioni}

II dialetto istroveneto parlato a livello autoctono nell'Istria slovena è un idioma alloctono importato in Istria dalla Repubblica di Venezia a partire dal $X$ secolo. La posizione sociolinguistica di questo dialetto mutò drasticamente nell'immediato dopoguerra - il dialetto che fino alla fine della Seconda guerra mondiale aveva avuto il ruolo di koinè di tutti gli istriani, nell'immediato dopoguerra perse all'incirca il novanta per cento dei suoi parlanti. All'annessione dell'Istria alla 
Repubblica popolare federativa di Jugoslavia seguirono l'esodo degli istriani di origini italiane e le ondate di nuovi arrivi provenienti da varie regioni slovene e dalle repubbliche jugoslave. Oggi l'istroveneto viene adoperato (quasi esclusivamente) dagli istriani di nazionalità italiana che risiedono lungo la fascia costiera e nell'immediato entroterra. Questo idioma convive da secoli con il dialetto di matrice slava, l'istrosloveno, conservatosi nell'entroterra di Capodistria, Isola e Pirano.

Le regole d'uso dei tempi verbali del modo indicativo esposte in questa sede sono state acquisite esclusivamente nel corso delle attività di ricerca sul campo, ovvero a Capodistria, Isola, Pirano, Strugnano, Sicciole, Crevatini e Bertocchi. Dalle ricerche è emerso che nell'odierno dialetto istroveneto gli elementi grammaticali di più antica derivazione si alternano ad altri più recenti, desunti dall'italiano. Dal materiale dialettale ricavato si evince per di più che il modo indicativo istroveneto conosce sette tempi verbali - il presente, l'imperfetto, il futuro semplice, il passato prossimo, trapassato prossimo, il futuro anteriore. Dall'analisi dei testi dialettali è emerso che con il tempo presente i dialettofoni istroveneti comunicano situazioni che si svolgono al momento dell'enunciazione, descrivono le consuetudini (anche usanze) del soggetto, indicano avvenimenti che devono ancora realizzarsi, raccontano un evento passato conclusosi (presente storico), enunciano il verificarsi di proprietà permanenti o eterne (presente atemporale), "eterne verità", proverbi ecc.

Con l'imperfetto indicano fondamentalmente la simultaneità rispetto ad un momento passato, descrivono azioni ripetute e continuative nel passato, segnalano azioni incompiute nel passato, descrivono azioni svoltesi nel passato e formulano frasi ipotetiche relative al periodo ipotetico dell'irrealtà. II tempo futuro istroveneto annuncia avvenimenti che devono ancora svolgersi o che si realizzeranno. L'istroveneto conosce altresi tre tempi verbali composti, formati dall'ausiliare 'eser 'essere' o ver 'avere' (al tempo addicente) e dal verbo al participio passato, ossia: il passato prossimo, il futuro anteriore e il trapassato prossimo. Mentre il tempo passato prossimo descrive avvenimenti conclusisi nel passato, il trapassato indica azioni anteriori rispetto ad un momento conclusosi nel passato. Con futuro anteriore $\mathrm{i}$ dialettofoni del dialetto preso in esame verbalizzano avvenimenti anteriori rispetto ad un'azione futura. 


\section{Bibliografia:}

Dardano, Maurizio e Pietro Trifone. Grammatica italiana con nozioni di Linguistica. Bologna: Zanichelli, 2013.

Delise, Antonio. Vocabolarietto del dialetto isolano. Trieste: Isola nostra, 2001.

Filipi, Goran. "Istriotski jezikovni otoki v Istri." Annales: Series Historia et Sociologia 11 (1993): 275-284.

--------- "Slovanske in predneolatinske jezikovne prvine $\mathrm{v}$ listini Rižanskega placita: (oziroma neuspel poskus določanja le-teh)." Acta Histriae 2 (1994): 25-30.

Manzini, Giuliano e Luciano Rocchi. Dizionario storico fraseologico etimologico del dialetto di Capodistria. Rovigno: Centro di ricerche storiche Rovigno, 1995.

Lusa, Ondina. Le perle del nostro dialetto. Pirano: Comunità degli Italiani »Giuseppe Tartini« Pirano, 2004.

Lusa, Ondina, Marino Bonifacio. Le perle del nostro dialetto. Vol. 2. Pirano: Comunità degli Italiani “Giuseppe Tartini” Pirano, 2010.

Lusa, Ondina, Marino Bonifacio, M. (2012): Le perle del nostro dialetto. Pirano: Comunità degli Italiani “Giuseppe Tartini” Pirano, 2012.

Sau, Silvano. Dizionario del dialetto Isolano: raccolta di parole e modi di dire della parlata isolana di ieri, di oggi e, forse, di domani. Isola: II Mandracchio, 2009.

Todorović, Suzana. Il dialetto istroveneto a Capodistria, Isola e Pirano. Capodistria: Libris, 2017.

La varietà dialettale del Capodistriano. Capodistria: Libris, 2018a.

Istroveneto nell'ambito degli altri idiomi delle città slovene costiere. Capodistria: Libris, 2019.

Istrskobeneški jezikovni atlas severozahodne Istre. 1, Vremenske razmere, geomorfologija, običaji in institucije, telo in bolezni/Atlante linguistico istroveneto dell'Istria nordoccidentale. 1, Fenomeni atmosferici, configurazione del terreno, tradizioni ed istituzioni, corpo e malattie. Capodistria: Unione Italiana, Libris, Biblioteca centrale Srečko Vilhar Capodistria, 2019a.

Parovel, Dino. Il dizionario del dialetto capodistriano. Trieste: Fameia capodistriana, 2006.

Šircelj, Milivoja. Verska, narodna in jezikovna sestava prebivalstva Slovenije; popisi 1921-2002. Ljubljana: Statistični urad republike Slovenije, 2003. 
Žitko, Salvator. Koper: mestne znamenitosti. Koper: Libris, 2011.

Fonti orali (informatori)

Bonifacio, M. (2015). Comunicazione orale.

Cernaz, A. (2015). Comunicazione orale.

Dellore, E. (2015). Comunicazione orale.

Filippi, P. (2015). Comunicazione orale.

Kalc, B. (2017). Comunicazione orale.

Moscolin, A. M. (2015). Comunicazione orale.

Novel, B. (2017). Comunicazione orale.

Novel, G. (2017). Comunicazione orale.

Novello, M. P. (2016). Comunicazione orale.

Rasman, P (2015). Comunicazione orale.

Scheriani, G. (2016). Comunicazione orale.

Scheriani, N. (2016). Comunicazione orale.

Tuljak, A. (2018). Comunicazione orale.

Zupin, N. (2017). Comunicazione orale.

\section{INDIKATIV GLAGOLSKIH VREMENA U ISTROMLETAČKOM DIJALEKTU SLOVENSKIH PRIMORSKIH MJESTA}

$\mathrm{U}$ članku pred nama raspravljamo o uporabi glagolskih vremena u istromletačkome u okviru talijanskoga indikativa.

Istromletački leksik sjeverozapadne Istre zapisan je u osam rječnika Dizionario storico fraseologico etimologico del dialetto di Capodistria (Giulio Manzini in Luciano Rocchi, 1995.), Dizionario del dialetto capodistriano (Dino Parovel, 2006), Voci della parlata isolana nella prima metà di questo secolo (Antonio Vascotto, 1987.), Vocabolarietto del dialetto isolano (Antonio Delise, 2006.), Dizionario del dialetto isolano: raccolta di parole e modi di dire della parlata isolana di ieri, di oggi e, forse, di domani (Silvano Sau, 2009.), Le perle del nostro dialetto (Ondina Lusa i Marino Bonifacio, 2004., 2010., 2012.). U nekim od spomenutih zabilježeni su i elementi gramatike, no oni se ne slažu uvijek s onim što smo sami čuli od dijalektofona. Valja naglasiti da su to zapisi prije svega domaćih ljudi, ljubitelja domaćega izričaja.

Pravila uporabe glagolskih vremena $\mathrm{u}$ istromletačkome (presente, imperfetto, futuro semplice, passato prossimo, imperfetto, trapassato prossimo, futuro anteriore) izdvojili smo iz tekstova u dijalektu koje 
smo snimili i zapisali u okviru opsežnih dijalektalnih istraživanja u Kopru, Izoli, Piranu, Strunjanu, Sečovlju, Hrvatinima i Bertokima. Tekstove smo dosljedno opremili prijevodom na talijanski. Za sva smo prikazana glagolska vremena zapisali konjugacije pomoćnih glagola 'eser 'biti' i ver 'imati' i konjugacije pravilnih glagola $s$ infinitivnim nastavcima -ar ili a- (Piran); -er (Kopar), -e (Izola) ili -i (Piran i Strunjan); -ir (Kopar, Izola) ili -i (Piran). Za složena glagolska vremena pokazali smo njihovu tvorbu.

Ključne riječi: glagolski način, indikativ, glagolska vremena, istromletački dijalekat, slovenska Istra, dijalektologija 